\title{
Nematode fauna of Rajaji National Park, with First record of Granonchulus subdecurrens Coetzee, 1966 (Mononchida: Mylonchulidae) from India
}

\author{
Vinita Sharma, Alka Dubey ${ }^{*}$ \\ Zoological Survey of India, Northern Regional Centre, Dehradun, Uttarakhand, India
}

Email address:

alkabioinfo964@gmail.com (A. Dubey) vinitascb@gmail.com (V. Sharma)

To cite this article:

Vinita Sharma, Alka Dubey. Nematode Fauna of Rajaji National Park, with First Record of Granonchulus subdecurrens Coetzee, 1966 (Mononchida: Mylonchulidae) from India. Agriculture, Forestry and Fisheries. Vol. 4, No. 1, 2015, pp. 1-6. doi: 10.11648/j.aff.20150401.11

\begin{abstract}
A total 26 species of terrestrial nematode (15 from order Dorylaimida and 11 from order Mononchida) has been recorded from Rajaji National Park (RNP), Uttarakhand, India. All are being reported first time from RNP. Of these, Granonchulus subdecurrens Coetzee, 1966 is being recorded from first time from India.
\end{abstract}

Keywords: Dorylaimida, Mononchida, Granonchulus, New Records, Rajaji National Park, India

\section{Introduction}

India is a rich faunal diversity country. In invertebrate fauna, nematode constitute second largest group after arthropods. Significant work on terrestrial nematode already being done in India by reporting 2100 species of nematodes from different ecosystem [9]. A number of Indian Nematologist $[1-3,5-7]$ made their contribution to work out nematode fauna of Protected areas viz., Keoladeo National Park, Kaziranga National Park, Rajaji National Park and Ranthambore National Park and Silent Valley National Park.

In Uttarakhand, Rajaji National Park (RNP) is one of the important conservation area, because it has rich bio-diversity. In nematode fauna only 5 species have been already recorded from by the present authors [5] from this protected area. In the present paper an account of identified 26 species of nematode from this protected area is presented, of which Granonchulus subdecurrens is first reported from country.

\section{Materials and Methods}

During survey for nematode fauna of RNP in 2013, soil samples were collected soil around the of forest tree species from Chilla Range, Jhabar and Hazra Beats, RNP, Haridwar, were processed by sieving and decantation and modified Baerman's funnel techniques. Extracted nematodes were heat-killed and fixed in hot $4 \%$ formalin. The nematodes will be transferred from fixative to a solution of 5 parts of glycerin and 95 parts of $30 \%$ alcohol in a cavity block and dehydrated by slow evaporation method [8] and mounted in anhydrous glycerin. The mounted nematodes were later measured by ocular micrometer and drawn using drawing tube attached to Olympus BX-51 DIC Microscope. LM photographs were taken using Olympus digital camera.

\section{Results}

During nematode survey 26 species has been recorded from RNP, belonging to 11 genera, 7 families and 2 orders, of Dorylaimida and Mononchida. Four species of Xiphinema are plant parasitic in nature.

All 26 species are recorded for first time from this protected area, of which Granonchulus subdecurrens Coetzee, 1966 is being reported from India. All the specimens are registered in National Zoological Collection, NRC, ZSI, Dehra Dun, India.

Order Dorylaimida

Family Aporcelaimidae Heyns, 1965

1. Aporcelaimellus capitatus (Thorne \& Swanger, 1936) Heyns, 1965

2. Aporcelaimellus clamus Throne, 1974

3. Aporcelaimellus invisus Tjepkema, Ferris and Ferris, 1971

4. Aporcelaimellus obscurus (Thorne \& Swanger, 1936) Heyns, 1965

Family Qudsianematidae Jairajpuri, 1965

5. Eudorylaimus chauhani (Baqri \& Khera, 1975) Andrassy, 1986 
6. Allodorylaimus irritans (Cobb in Thorne \& Swanger, 1936) Andrassy, 1986

7. Discolaimus major Thorne, 1939

8. Discolaimus tenax Siddiqi, 1964

9. Discolaimus texanus Cobb, 1913

10. Discolaimus similis Thorne, 1939

Family Xiphinematidae Dalmasso, 1969

11. Xiphinema americanum Cobb, 1913

12. Xiphinema inaequle Khan \& Ahmad, 1974

13. Xiphinema insigne Loos, 1949

14. Xiphinema opisthosternum Siddiqi, 1961

Family Mydonomidae Thorne, 1964

15. Dorylaimoides micoletzkyi (de Man, 1921) Thorne \& Swanger, 1936

Order Mononchida Jairajpuri, 1969

Family Mylonchulidae Jairajpuri, 1969

16. Mylonchulus armus Khan \& Jairajpuri, 1979

17. Mylonchulus brachyurus (Butschli, 1873) Andrassy, 1958

18. Mylonchulus hawaiensis (Cassidy, 1931) Andrassy, 1958

19. Paramylonchulus mashhoodi (Khan \& Jairajpuri, 1979) Jarajpuri \& Khan, 1981

20. Paramylonchulus mulveyi (Khan \& Jairajpuri, 1979) Jarajpuri \& Khan, 1981

21. Granonchulus subdecurrens Coetzee, 1966

Family Iotonchidae Jairajpuri, 1969

22. Iotonchus indicus Jairajpuri, 1969

23. Iotonchus parabasiodontus Mulvey \& Jensen, 1967

24. Iotonchus trichurus(Cobb, 1917) Andrassy, 1958

25. Iotonchulus longicaudatus (Baqri, Baqri \& Jairajpuri, 1978) Andrassy, 1993

Family Bathyodontidae Clark, 1961

26. Bathyodontus mirus(Andrassy, 1956) Andrassy in Hooper \& Cairns, 1959

\section{Abbreviations}

$\mathrm{L}=$ Total body length; $\mathrm{a}=$ Body length/greatest body width; $b=$ Body length/neck length; $c=$ Body length/tail length; $c^{\prime}=$ Tail length/body width at anus; $\mathrm{V}=$ Distance of vulva from ant. end x 100/body length

\section{Description and Diagnosis}

1. Aporcelaimellus capitatus (Thorne \& Swanger, 1936) Heyns, 1965

Morphological taxonomic calculation: Female: $\mathrm{L}=2.0 \mathrm{~mm}$; $\mathrm{a}=27 ; \mathrm{b}=3.7 ; \mathrm{c}=53 ; \quad \mathrm{c}^{\prime}=1.1 ; \mathrm{V}=54 ; \quad$ Odontostyle $=20 \mu \mathrm{m}$; Odontophore $=40 \mu \mathrm{m}$

Description: Female: Body ventrally arcuated upon fixtation. Lip region discoid. Lips with prominent papillae. Vulva a transverse slit. Reproductive system amphidelphic. Tail dorsally convex.

Male: Not found.

Locality and habitat: Soil around the roots of Shorea robusta.

2. Aporcelaimellus clamus Throne, 1974
Morphological taxonomic calculation: Female: $\mathrm{L}=2.3 \mathrm{~mm}$; $\mathrm{a}=29 ; \mathrm{b}=4.0 ; \mathrm{c}=59 ; \mathrm{c}^{\prime}=0.9 ; \mathrm{V}=53 ;$ Odontostyle $=18 \mu \mathrm{m}$; Odontophore $=38 \mu \mathrm{m}$

Description: Female: Body slightly arcuate upon fixation. Lips region set off by slightly narrowing of neck. Vulva a transverse slit. Reproductive system amphidelphic. Tail bluntly conoid.

Male: Not found.

Locality and habitat: Soil around the roots of Shorea robusta from Hazra Beat.

3. Aporcelaimellus invisus Tjepkema, Ferris and Ferris, 1971

Morphological taxonomic calculation: Female: $\mathrm{L}=2.1 \mathrm{~mm}$; $\mathrm{a}=26 ; \mathrm{b}=3.7 ; \mathrm{c}=55 ; \quad \mathrm{c}^{\prime}=0.9, \quad \mathrm{~V}=54 ; \quad$ Odontostyle $=20 \mu \mathrm{m}$; Odontophore $=40 \mu \mathrm{m}$.

Description: Female: Body slightly arcuate upon fixation. Lips region set off by slightly narrowing of neck. Odontostyle aperture $63 \%$ of odontostyle length. Vulva a transverse slit. Reproductive system amphidelphic. Tail long, bluntly conoid.

Male: Not found.

Locality and habitat: Soil around the roots of unidentified trees from Hazra Beat.

Remarks: The measurements of present specimens fit well with the specimen described by Tjepkema, Ferris and Ferris, 1971 except odontophore (length obscure in type).

4. Aporcelaimellus obscurus(Thorne \& Swanger, 1936) Heyns, 1965

Morphological taxonomic calculation: Female: $\mathrm{L}=2.2 \mathrm{~mm}$; $\mathrm{a}=28 ; \mathrm{b}=3.6 ; \mathrm{c}=68 ; \mathrm{c}=0.76, \mathrm{~V}=58 ; \quad$ Odontostyle $=21 \mu \mathrm{m}$; Odontophore $=40 \mu \mathrm{m}$

Description: Female: Body ventrally arcuated upon fixtation. Lips distinct, well separated. Vulva a transverse slit. Reproductive system amphidelphic. Tail convex-conoid, tip blunt.

Male: Not found.

Locality and habitat: Soil around the roots of Ehretia laevis from Jhabar Beat.

5. Eudorylaimus chauhani (Baqri \& Khera, 1975) Andrassy, 1986

Morphological taxonomic calculation: Female: $\mathrm{L}=1.5 \mathrm{~mm}$; $\mathrm{a}=29 ; \quad \mathrm{b}=4.0 ; \quad \mathrm{c}=24 ; \quad \mathrm{c}^{\prime}=2.3 ; \quad \mathrm{V}=50 ; \quad$ Odontostyle $=18 \mu \mathrm{m}$; Odontophore $=30 \mu \mathrm{m}$

Description: Female: Body curved ventrally upon fixation. Lip region well offset by constriction. Vulva a transverse slit. Reproductive system amphidelphic. Tail dorsally convexconoid with sub-acute tip.

Male: Not found.

Locality and habitat: Soil around the roots of Tectona grandis from Hazra Beat.

1. Allodorylaimus irritans (Cobb in Thorne \& Swanger, 1936) Andrassy, 1986

Morphological taxonomic calculation: Female: $\mathrm{L}=1.2 \mathrm{~mm}$; $\mathrm{a}=23 ; \mathrm{b}=3.7 ; \quad \mathrm{c}=27 ; \quad \mathrm{c}^{\prime}=1.8 ; \mathrm{V}=51 ; \quad$ Odontostyle $=20 \mu \mathrm{m}$; Odontophore $=45 \mu \mathrm{m}$

Description: Female: Body curved ventrally upon fixation. Lip region offset. Lips well separated. Odontostyle aperture 
is $50 \%$ of odontostyle length. The expanded part of the oesophagus occupies 1/3 of total oesophageal length. Vulva a transverse slit. Reproductive system amphidelphic. Tail dorsally convex and tail tip acute.

Male: Not found.

Locality and habitat: Soil around the roots of unidentified plants from Hazra Beat.

2. Discolaimus major Thorne, 1939

Morphological taxonomic calculation: Female: $\mathrm{L}=2.2 \mathrm{~mm}$; $\mathrm{a}=37 ; \quad \mathrm{b}=4.4 ; \quad \mathrm{c}=55 ; \quad \mathrm{c}^{\prime}=1.2, \quad \mathrm{~V}=50 ; \quad$ Odontostyle $=21 \mu \mathrm{m}$; Odontophore $=43 \mu \mathrm{m}$

Description: Female: Body slightly curved ventrally upon fixation. Lip region discoid, set off from body. Vulva a transverse slit. Reproductive system amphidelphic. Tail dorsally convex-conoid, with rounded tip.

Male: Not found.

Locality and habitat: Soil around the roots of Shorea robusta from Chilla Range.

3. Discolaimus similis Thorne, 1939

Morphological taxonomic calculation: Female: $\mathrm{L}=1.3 \mathrm{~mm}$;

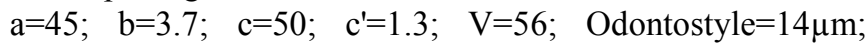
Odontophore $=20 \mu \mathrm{m}$

Description: Female: Body slightly curved ventrally upon fixation. Lip region discoid, set off from body. Odontostyle aperture $54 \%$ of odontostyle length. Vulva a transverse slit. Reproductive system amphidelphic. Tail dorsally convexconoid, with rounded tip.

Male: Not found.

Locality and habitat: Soil around the roots of Ficus benghalensis from Hazra Beat.

4. Discolaimus tenax Siddiqi, 1964

Morphological taxonomic calculation: Female: $\mathrm{L}=1.5 \mathrm{~mm}$; $\mathrm{a}=39 ; \mathrm{b}=4.5 ; \mathrm{c}=53 ; \mathrm{c}^{\prime}=1.5 ; \mathrm{V}=54 ;$ Odontostyle $=16 \mu \mathrm{m}$; Odontophore $=26 \mu \mathrm{m}$

Description: Female: Body ventrally arcuate upon fixation. Lip region set off by constriction. Vulva a transverse slit. Reproductive system amphidelphic. Tail convex conoid, rounded.

Locality and habitat: Soil around the roots of Dalbergia sissoo from Chilla Range.

5. Discolaimus texanus Cobb, 1913

Morphological taxonomic calculation: Female: $\mathrm{L}=1.2 \mathrm{~mm}$; $\mathrm{a}=41 ; \mathrm{b}=3.9 ; \quad \mathrm{c}=46 ; \quad \mathrm{c}^{\prime}=1.2 ; \quad \mathrm{V}=37 ; \quad$ Odontostyle $=14 \mu \mathrm{m}$; Odontophore $=28 \mu \mathrm{m}$

Description: Female: Body ventrally arcuate upon fixation. Lip region set off by constriction. Vulva a transverse slit. Reproductive system amphidelphic. Tail convex conoid, rounded.

Male: Not found.

Locality and habitat: Soil around the roots of Terminalia bellirica Roxb. from Hazara Beat.

6. Xiphinema americanum Cobb, 1913

Morphological taxonomic calculation: Females: $\mathrm{L}=1.6-$ $1.9 \mathrm{~mm} ; \mathrm{a}=46-50 ; \mathrm{b}=5.2-5.7 ; \mathrm{c}=42-63 ; \mathrm{c}^{\prime}=1.4-1.9 ; \mathrm{V}=50-55$; Guiding $\quad$ ring $=70-75 \mu \mathrm{m}$; $\quad$ Odontostyle $=75-88 \mu \mathrm{m}$; Odontophore $=48-53 \mu \mathrm{m}$; Prerectum $=143 \mu \mathrm{m}$
Description: Females: Body C shaped upon fixation. Lip region rounded and set off from body. Amphid stirrup shaped with slit like apertures and located at the base of lip region. Basal bulb of oesophagus 23-25\% of the length. Reproductive system amphidelphic. Tail short, convexconoid.

Male: Not found.

Locality and habitat: Soil around the roots of Dalbergia sissoo from Chilla Range.

7. Xiphinema inaequle Khan \& Ahmad, 1974

Morphological taxonomic calculation: Females: L=2.2$2.4 \mathrm{~mm} ; \quad \mathrm{a}=65-66 ; \quad \mathrm{b}=5.9-6.0 ; \quad \mathrm{c}=26-29 ; \quad \mathrm{c}^{\prime}=3.8 ; \quad \mathrm{V}=28-3$; Guiding ring $=100 \mu \mathrm{m}$; Odontostyle $=108 \mu \mathrm{m}$; Odontophore $=$ $58 \mu \mathrm{m}$; Prerectum $=250 \mu \mathrm{m}$

Description: Females: Body $\mathrm{C}$ shaped upon fixation. Lip region rounded almost continuous with body. Amphid stirrup shaped with slit like apertures. Basal bulb of oesophagus 23$25 \%$ of the length. Reproductive system amphidelphic. Tail short conoid,

Male: Not found.

Locality and habitat: Soil around the roots of Shorea robusta and Dalbergia sissoo from Chilla Range.

8. Xiphinema insigne Loos, 1949

Morphological taxonomic calculation: Females: $\mathrm{L}=2.1$ 2.4mm; $a=64-66 ; b=5.9-6.0 ; c=23-29 ; c^{\prime}=3.7-3.9 ; V=28-29$; Guiding ring $=88-100 \mu \mathrm{m} ; \quad$ Odontostyle $=108-118 \mu \mathrm{m}$; Odontophore $=58-63 \mu \mathrm{m}$; Prerectum $=386-540 \mu \mathrm{m}$

Description: Females: Body almost slightly curved upon fixation Lip region almost flat or rounded and slightly set off from body. Amphid stirrup shaped with slit like apertures and located at the base of lip region. Basal bulb of oesophagus $22-25 \%$ of the length. Reproductive system amphidelphic. Tail narrow, elongate conoid,

Male: Not found.

Locality and habitat: Soil around the roots of Trewia nudiflora Linn from Chilla Range.

9. Xiphinema opisthosternum Siddiqi, 1961

Morphological taxonomic calculation: Female: $\mathrm{L}=1.4 \mathrm{~mm}$; $\mathrm{a}=61 ; \mathrm{b}=5.4 ; \mathrm{c}=43 ; \mathrm{c}^{\prime}=2.3 ; \mathrm{V}=67 ;$ Guiding ring $=56 \mu \mathrm{m}$; Odontostyle $=69 \mu \mathrm{m}$; Odontophore $=38 \mu \mathrm{m}$

Description: Female: Body C shaped upon fixation. Lip region rounded and set off from body. Amphid stirrup shaped. Basal bulb of oesophagus $22 \%$ of the length Reproductive system amphidelphic. Tail elongate-conoid.

Male: Not found.

Locality and habitat: Soil around the roots of Dalbergia sissoo from Chilla Range.

10. Dorylaimoides micoletzkyi (de Man, 1921) Thorne \& Swanger, 1936

Morphological taxonomic calculation: Female: $\mathrm{L}=1.3 \mathrm{~mm}$; $\mathrm{a}=36 ; \mathrm{b}=6.3 ; \mathrm{c}=20 ; \mathrm{V}=40$; Odontostyle $=8 \mu \mathrm{m}$

Description: Female: Body cylindroids arcuate upon fixation. Head slightly constricted, lips smooth. Amphids cup shaped. Odontostyle dorylaimoid, arcuate. Odontophore arcuate, Reproductive system amphidelphic. Tail elongate conoid, terminus dorsally bent.

Male: Not found. 
Locality and habitat: Soil around the roots of Bahunia varigata from Chilla Range.

11. Mylonchulus armus Khan \& Jairajpuri, 1979

Morphological taxonomic calculation: Female: $\mathrm{L}=81 \mathrm{~mm}$; $\mathrm{a}=29 ; \mathrm{b}=3.1 ; \mathrm{c}=25 ; \mathrm{c}^{\prime}=1.8 ; \mathrm{V}=58$

Description: Female: Lip region $18 \mu \mathrm{m}$ x $8 \mu \mathrm{m}$. Buccal cavity $20 \mu \mathrm{m} \times 13 \mu \mathrm{m}$. Dorsal tooth of median size, its apex at $15 \mu \mathrm{m}$. from base of buccal cavity. Subventral walls with 5 transverse rows of denticles. Submedian teeth absent. Reproductive system amphidelphic. Tail conoid with clavate terminus. Caudal glands grouped. Spinneret terminal.

Male: Not found.

Locality and habitat: Soil around the roots of Ehretia laevis Roxb.from Jhabar Beat.

12. M. brachyurus (Butschli, 1873) Andrassy, 1958

Morphological taxonomic calculation: Female: $\mathrm{L}=1.0 \mathrm{~mm}$; $\mathrm{a}=22 ; \mathrm{b}=3.0 ; \mathrm{c}=20 ; \mathrm{c}^{\prime}=2.2 ; \mathrm{V}=60 \%$

Description: Female: Lip region $25 \mu \mathrm{m}$ x $10 \mu \mathrm{m}$. Buccal cavity $22 \mu \mathrm{m} \times 14 \mu \mathrm{m}$. Dorsal tooth of massive, its apex at $15 \mu \mathrm{m}$ from base of buccal cavity. Subventral walls with 6 transverse rows of denticles. Submedian teeth present. Reproductive system amphidelphic. Tail conoid with blunt terminus. Caudal glands grouped. Spinneret subterminal.

Male: Not found.

Locality and habitat: Soil around the roots of Tectona grandis from Chilla Range.

13. M. hawaiensis (Cassidy, 1931) Andrassy, 1958

Morphological taxonomic calculation: Females: $\mathrm{L}=0.82-$ $0.86 \mathrm{~mm} ; \mathrm{a}=23-27 ; \mathrm{b}=3.2-3.3 ; \mathrm{c}=19-29 ; \mathrm{c}^{\prime}=1.4-1.9 ; \mathrm{V}=58$ 59

Description: Females: Lip region $22-29 \mu \mathrm{m}$ x 5-10 $\mu \mathrm{m}$. Buccal cavity $16-23 \mu \mathrm{m} \times 10-14 \mu \mathrm{m}$. Dorsal tooth of massive, its apex at $13-15 \mu \mathrm{m}$ from base of buccal cavity. Subventral walls with 7 transverse rows of denticles. Submedian teeth present. Reproductive system amphidelphic. Tail tip slightly clavate. Caudal glands tandem. Spinneret terminal.

Male: Not found.

Locality and habitat: Soil around the roots of Shorea robusta from Chilla Range.

14. Paramylonchulus mashhoodi (Khan \& Jairajpuri, 1979) Jarajpuri \& Khan, 1981

Morphological taxonomic calculation: Female: $\mathrm{L}=1.1 \mathrm{~mm}$; $\mathrm{a}=37 ; \mathrm{b}=3.3 ; \mathrm{c}=23 ; \mathrm{c}^{\prime}=2.1 ; \mathrm{V}=76$

Description: Female: Lip region $20 \mu \mathrm{m}$ x $5 \mu \mathrm{m}$. Buccal cavity $18 \mu \mathrm{m} \times 11 \mu \mathrm{m}$. Dorsal tooth of median size, its apex at $15 \mu \mathrm{m}$ from base of buccal cavity. Subventral walls with 3 or 4 transverse rows of denticles. Submedian teeth absent. Reproductive system mono-prodelphic. Posterior uterine sac absent. Tail conoid, tapering sharply in posterior half, with a slightly rounded tip. Caudal glands grouped. Spinneret terminal.

Male: Not found.

Locality and habitat: Soil around the roots of Cassia fistula Linn. from Hazra Beat.

15. Paramylonchulus mulveyi (Khan \& Jairajpuri, 1979) Jarajpuri \& Khan, 1981

Morphological taxonomic calculation: Female: $\mathrm{L}=1.0 \mathrm{~mm}$; $\mathrm{a}=34 ; \mathrm{b}=3.2 ; \mathrm{c}=21 ; \mathrm{c}^{\prime}=2.1 ; \mathrm{V}=78$

Description: Female: Lip region $20 \mu \mathrm{m}$ x $8 \mu \mathrm{m}$. Buccal cavity $15 \mu \mathrm{m} \times 9 \mu \mathrm{m}$. Dorsal tooth of massive, its apex at $14 \mu \mathrm{m}$ from base of buccal cavity. Subventral walls with 4 transverse rows of denticles. Submedian teeth absent. Reproductive system mono-prodelphic. Posterior uterine sac absent. Tail elongate conoid. Caudal glands grouped. Spinneret terminal.

Male: Not found.

Locality and habitat: Soil around the roots of Cordia oblicua from Hazra Beat.

16. Granonchulus subdecurrens Coetzee(1966)

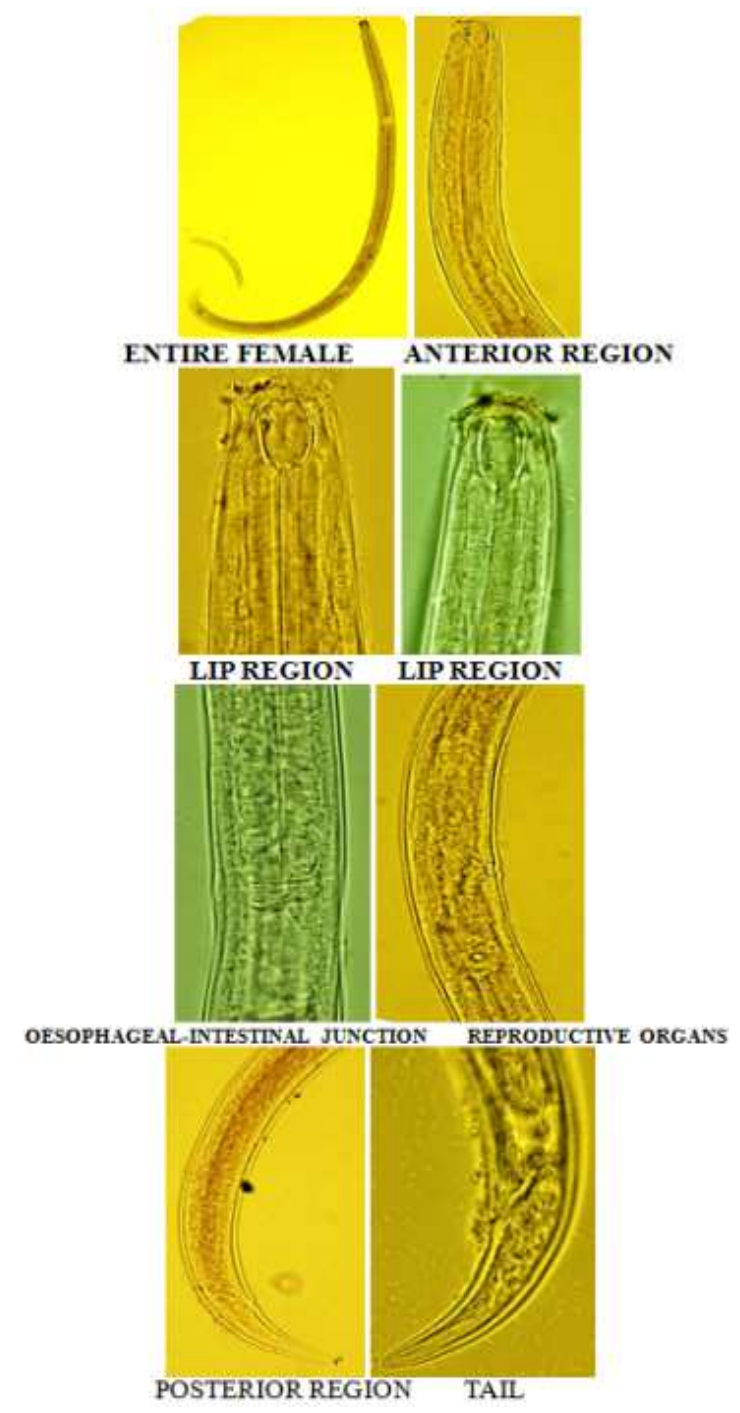

Fig 1. Female Granonchulus subdecurrens

Morphological taxonomic calculation: Females: $\mathrm{L}=1.3-$ 1.4mm; $a=33-37 ; b=4.3-4.5 ; c=19-21 ; c^{\prime}=2.7, V=59$

Description: Females: Body ventrally arcuated upon fixtation. Lip region slightly offset, bearing papillae, 23$24 \mu \mathrm{m} \times 9-13 \mu \mathrm{m}$. Buccal cavity barrel shaped, $21-22 \mu \mathrm{m} \times 11-$ $13 \mu \mathrm{m}$. Amphid cup shaped, situated slightly anterior to dorsal tooth apex; aperture slit like, about $4 \mu \mathrm{m}$ wide. Dorsal tooth moderately developed, anteriorly developed, its apex at 
$63-67 \%$ of the buccal cavity length from its base, sub ventral small denticle, opposed to dorsal tooth, arranged in two groups, an anterior transverse row in line with the dorsal tooth apex and posterior irregular arranged group extending to base of stoma, oesophagus-intestinal junction nontuberculated. Reproductive system amphidelphic. Valve transverse slit, vagina sclerotized. Pre-rectum absent. Tail conoid, ventrally arcuated with rounded tip. Caudal glands three, arranged in tandem, opening terminal.

Male: Not found.

Locality and habitat: Soil around the roots of Shorea robusta from Chilla Range.

Distribution: South Africa.

Remarks: This species was originally described from South Africa (Coetzee, 1966) and has been recorded thereafter. The description given agrees well with original description.

This is the first report from India and after original its description.

17. Iotonchus indicus Jairajpuri, 1969

Morphological taxonomic calculation: Female: $\mathrm{L}=2.1 \mathrm{~mm}$; $\mathrm{a}=29 ; \mathrm{b}=4.6 ; \mathrm{c}=6 ; \mathrm{c}^{\prime}=9 ; \mathrm{V}=58$

Description: Female: Lip region $45 \mu \mathrm{m}$ x $15 \mu \mathrm{m}$. Buccal cavity $45-55 \mu \mathrm{m} \times 30 \mu \mathrm{m}$. Dorsal tooth small and basal, its apex at $9 \mu \mathrm{m}$ from base of buccal cavity. Subventral walls with 4 transverse rows of denticles. Submedian teeth absent. Reproductive system amphidelphic. Tail elongate conoid. Caudal glands poorly developed. Opening subterminal dorsally.

Male: Not found.

Locality and habitat: Soil around the roots of Tectona grandis from Chilla Range.

18. Iotonchus parabasiodontus Mulvey \& Jensen, 1967

qMorphological taxonomic calculation: Female: $\mathrm{L}=1.6 \mathrm{~mm}$; $\mathrm{a}=40 ; \mathrm{b}=4.6 ; \mathrm{c}=3.6 ; \mathrm{c}^{\prime}=18 ; \mathrm{V}=59$

Description: Female: Lip region $26 \mu \mathrm{m}$ x $5 \mu \mathrm{m}$. Buccal cavity $26 \mu \mathrm{m} \times 17 \mu \mathrm{m}$. Dorsal tooth minute and basal, its apex at $7 \mu \mathrm{m}$ from base of buccal cavity. Submedian teeth absent. Reproductive system prodelphic. Tail conoid than cylindroids. Caudal glands present. Opening terminal.

Male: Not found.

Locality and habitat: Soil around the roots of Bahunia varigata from Chilla Range.

19. Iotonchus trichurus(Cobb, 1917) Andrassy, 1958

Morphological taxonomic calculation: Female: $\mathrm{L}=1.6 \mathrm{~mm}$; $\mathrm{a}=39 ; \mathrm{b}=5.3 ; \mathrm{c}=4 ; \mathrm{c}^{\prime}=17 ; \mathrm{V}=58$

Description: Female: Lip region $28 \mu \mathrm{m}$ x $10 \mu \mathrm{m}$. Buccal cavity $30 \mu \mathrm{m} \times 18 \mu \mathrm{m}$. Dorsal tooth small and basal, its apex at $8 \mu \mathrm{m}$ from base of buccal cavity. Subventral walls with 4 transverse rows of denticles. Submedian teeth absent. Reproductive system mono-prodelphic. Tail long. Caudal glands present. Terminal opening present.

Male: Not found.

Locality and habitat: Soil around the roots of Ficus religiosa Linn. from Jhabar Beat.

20. Iotonchulus longicaudatus (Baqri, Baqri \& Jairajpuri, 1978) Andrassy, 1993
Morphological taxonomic calculation: Female: $\mathrm{L}=1.3 \mathrm{~mm}$; $\mathrm{a}=37 ; \mathrm{b}=4.5 ; \mathrm{c}=3.8 ; \mathrm{c}^{\prime}=15 ; \mathrm{V}=59$

Description: Female: Lip region $25 \mu \mathrm{m}$ x $15 \mu \mathrm{m}$. Buccal cavity $18 \mu \mathrm{m} \times 10 \mu \mathrm{m}$. Dorsal tooth medium size, in anterior half of buccal cavity, its apex at $19 \mu \mathrm{m}$ from base of buccal cavity. Reproductive system mono-prodelphic. Tail long, filiform. Caudal glands present. Terminal opening present.

Male: Not found.

Locality and habitat: Soil around the roots of Shorea robusta from Chilla Range.

21. Bathyodontus mirus(Andrassy, 1956) Andrassy in Hooper \& Cairns, 1959

Morphological taxonomic calculation: Female: $\mathrm{L}=1.1 \mathrm{~mm}$; $\mathrm{a}=34 ; \mathrm{b}=3.5 ; \mathrm{c}=45 ; \mathrm{c}^{\prime}=0.8 ; \mathrm{V}=57$

Description: Female: Lip region $23 \mu \mathrm{m}$ x $5 \mu \mathrm{m}$, set off by a deep constriction, lips rounded. Buccal cavity $20 \mu \mathrm{m} \times 6 \mu \mathrm{m}$. Reproductive system amphidelphic. Tail rounded. Caudal glands present. Spinneret terminal.

Male: Not found.

Locality and habitat: Soil around the roots of unidentified tree from Chilla Range.

\section{Discussion}

The present authors have already reported five species of Mylonchulus from RNP [5]. Taking into account 5 already reported species, total terrestrial nematode species is now 31 .

Granonchulus helicus was recorded from Bareilly, Uttar Pradesh, India [10]. Granonchulus subdecurrens Coetzee, 1966 is second species recorded from India.

\section{Acknowledgements}

The authors wish to thanks Director, Zoological Survey of India, Kolkata and the Officer-in-Charge, NRC, ZSI, Dehradun for proving necessary facilities and authors also grateful for Uttarakhand State Council for Science and Technology, Dehradun, Uttarakhand, India for funding this scientific research project.

\section{References}

[1] W. Ahmad, Md. Banyamuddin and U. Tauheed, Rhinodorylaimus kazirangus gen. n., sp. n. (Dorylaimida: Dorylaimidae) from Kaziranga National Park, Assam, India, Nematology, 12, 2010, pp. 149-155.

[2] Md. Baniyamuddin and W. Ahmad, Two new and a known species of dorylaim nematodes (Dorylaimida: Nematoda) from Kaziranga National Park, Assam, India 45, 2011, pp. 2965-2980.

[3] P. Bohra and Q.H. Baqri, Plant and soil Nematodes from Ranthambore National Park, Rajasthan, India, Zoos Print Journal, 22, 2005, p. 2126.

[4] V. Coetzee, "Species of the genera Granonchulus and Cobbonchus (Mononchidae), occurring in Southern Africa," Nematologica 12, 1966, pp. 302-312. 
[5] A. Dubey and V. Sharma. Morphological Studies of Five Known Nematode Species Via Taxa-Informatics Approaches. Science Innovation. Vol. 2, No. 1, 2014, pp. 7-10. doi: 10.11648/j.si.20140201.12.

[6] R. Khan, A. Husain, R. Sultana and Q. Tahseen, Description of two new Monohystrid species, (Nematoda) from Keoladeo National Park, Rajasthan, India, Nematode medit., 33, 2005, pp. 67-73.

[7] T. Nusrat, A. Anjum and W. Ahmad, Mononchida (Nematoda) from Silent Valley National Park, India, Zootaxa 6535, 2013, pp. 224-236.
[8] J.W. Seinhorst, A rapid method for transfer of nematodes from fixative to anhydrous glycrine. Nematologica, 1959, 117-128.

[9] V. Sharma and Q.H. Baqri, Plant and Soil Nematodes of India: A Checkist. Bishen Singh Mahinder Pal Singh, Dehradun,2014, pp 266.

[10] R. K.Sharma and V. Saxena, "Granonchulus helicus sp.n. (Nematoda : Mononchida) from north India, Nematol. medit. 9, 1981, pp. 159-162. 